\title{
Vasculogenic mimicry in malignant mesothelioma: an experimental and immunohistochemical analysis
}

\author{
Emily Pulford ${ }^{1, *}$, Ashleigh Hocking ${ }^{1, *}$, Kim Griggs $^{1}$, James McEvoy $^{1}$, \\ Claudine Bonder $^{2}$, Douglas W. Henderson ${ }^{1,3}$ and Sonja Klebe ${ }^{1,3}$ \\ ${ }^{1}$ Department of Anatomical Pathology, Flinders University, ${ }^{2}$ Centre for Cancer Biology, \\ University of South Australia, SA Pathology, and School of Medicine, University of Adelaide, \\ and ${ }^{3}$ SA Pathology at Flinders Medical Centre, Bedford Park, SA, Australia; * these authors \\ contributed equally
}

\begin{abstract}
Summary
Vasculogenic mimicry, the process in which cancer cells form angiomatoid structures independent of or in addition to host angiogenesis has been recorded in several otherwise non-endothelial malignant neoplasms. This study describes evidence of routine vascular mimicry by human mesothelioma cell lines in vitro, when the cell lines are cultured alone or co-cultured with human umbilical vascular endothelial cells, with the formation of angiomatoid tubular networks. Vasculogenic mimicry is also supported by immunohistochemical demonstration of human-specific anti-mitochondria antibody labelling of tumour-associated vasculature of human mesothelioma cells xenotransplanted into nude mice, and by evidence of vascular mimicry in some biopsy samples of human malignant mesotheliomas. These studies show mosaic interlacing of cells that co-label or label individually for immunohistochemical markers of endothelial and mesothelial differentiation. If vascular mimicry in mesothelioma can be characterised more fully, this may facilitate identification of more specific and targeted therapeutic approaches such as anti-angiogenesis in combination with chemotherapy and immunotherapy or other therapeutic approaches.
\end{abstract}

Key words: Mesothelioma; vasculogenic mimicry; angiogenesis; VEGF; CD31; mesothelial markers.

Received 6 May, revised 11 July, accepted 18 July 2016

Available online 29 October 2016

\section{INTRODUCTION}

Malignant mesothelioma (MM) is an aggressive malignancy of the serosal membranes lining the pleural, peritoneal and pericardial cavities. Most MMs are attributable to asbestos exposure. Although there are now bans on asbestos in most industrialised countries, the incidence of pleural MM is stable or even increasing, due to long latency between commencement of exposure and diagnosis, and the continued presence of asbestos in the built environment. ${ }^{1-3}$ Prognosis remains poor, with median survivals of about 12 months, highlighting the importance of novel treatment strategies. ${ }^{4}$

Adequate blood supply is essential for tumour growth, invasion and metastasis. ${ }^{5}$ In mesothelioma, microvessel density is independently associated with decreased survival, ${ }^{5-7}$ and high levels of VEGF-A in serum and pleural effusions are adverse prognostic factors. ${ }^{8,9}$ Traditionally, tumour vascularisation was thought to occur via angiogenesis by the sprouting of pre-existing host blood vessels to form new vascular channels, but tumour angiogenesis is now thought to involve alternative mechanisms of vascularisation including vasculogenic mimicry (VM). First described in aggressive uveal melanomas, ${ }^{10}$ some tumour cells re-differentiate to an endothelium-like phenotype to form hollow channels and narrow conduits with a lumen through which blood can flow. ${ }^{11-15} \mathrm{VM}$ has been observed in other tumours, ${ }^{16-23}$ sometimes associated with decreased survival. ${ }^{19}$ The occurrence and significance of VM in MM has yet to be evaluated although there exists one report of VM in 'mesothelial sarcomas'. 24

In solid tumours, angiogenesis is stimulated by proangiogenic factors in response to hypoxia in the tumour microenvironment. Members of the VEGF family are important regulators of angiogenesis. ${ }^{8,9,25,26}$ There are several VEGF isoforms. ${ }^{27}$ VEGF-A and VEGF-B act via their receptors VEGFR1 (Flt-1) and VEGFR2 (Flk-1/KDR). ${ }^{28} \mathrm{MMs}$ secrete VEGF-A and can express VEGFR1, and in some MM cell lines VEGF-A acts directly as an autocrine growth factor in vitro, in a dose-dependent manner. ${ }^{29}$ This effect may be dependent on expression of VEGFR $1 .{ }^{30}$ Our observations suggest that although classical pathways of angiogenesis contribute to disease progression in $\mathrm{MM}$, alternative mechanisms of cancer progression such as VM may be significant.

$\mathrm{MM}$ is a morphologically protean tumour with epithelial, biphasic and sarcomatoid histological subtypes ${ }^{31-34}$ and, occasionally, heterologous sarcomatous differentiation such as chondroid and osseous differentiation. ${ }^{35}$ MMs routinely co-express epithelial and mesenchymal markers, such as cytokeratins and thrombomodulin, a glycoprotein expressed by mesothelium, vascular endothelium, synovium and placental syncytiotrophoblast. ${ }^{32,36-40}$ Sarcomatoid features predict poor prognosis, and the morphology-based MM classification correlates with expression profiles of molecular markers of epithelial-to-mesenchymal transition (EMT). ${ }^{41,42}$ Based on our clinical observations that rare MMs express specific vascular endothelial markers such as CD31 while maintaining cytokeratin expression and positive expression of mesothelial markers, and coupled with the limited clinical response to anti-angiogenic therapies, we postulated that MM is capable of VM. 


\section{MATERIALS AND METHODS}

\section{Mesothelioma cell cultures}

NCI-H226, NCI-H28, NCI-H2052, NCI-2452, MSTO-211H and Met5a cell lines were obtained from the ATCC and used within 20 passages. All cell lines were maintained in complete DMEM $(10 \%$ fetal calf serum, $50 \mathrm{U} / \mathrm{mL}$ penicillin, and $50 \mu \mathrm{g} / \mathrm{mL}$ streptomycin) and incubated at $37^{\circ} \mathrm{C}$ at $5 \% \mathrm{CO}_{2}$. Human umbilical vein endothelial cells (HUVECs) used as positive controls for tube formation assays were obtained from consenting donors ${ }^{43}$ (approved by the Human Research Ethics Committee of the Royal Adelaide Hospital, Adelaide, South Australia); HUVECs were cultured in M199 media supplemented with $20 \% \mathrm{FCS}, 50 \mathrm{U} / \mathrm{mL}$ penicillin, $50 \mu \mathrm{g} / \mathrm{mL}$ streptomycin, $1 \mu \mathrm{M}$ sodium pyruvate, $1 \% \mathrm{v} / \mathrm{v}$ non-essential amino acids and $1 \% \mathrm{v} / \mathrm{v}$ GlutaMAX.

\section{Harvesting of primary MM cells from pleural effusion fluids}

Twelve MM pleural effusion samples (with corresponding confirmatory biopsies), originating from eight patients ( 6 male, 2 female; 7 epithelioid and 1 sarcomatoid MM) were tested after diagnostic procedures were finalised (approved by Southern Adelaide Clinical Human Research Ethics Committee). Four benign reactive effusions were also tested. Samples were centrifuged at $500 \times \mathrm{g}$ for $10 \mathrm{~min}$ at $25^{\circ} \mathrm{C}$, and the cell pellet was cultured in complete DMEM. Supernatants were stored at $-80^{\circ} \mathrm{C}$. Purity of samples ( $>80 \%$ ) was confirmed by positive immunohistochemistry for the mesothelial cell marker calretinin and with the epithelial antibody CAM5.2. Diagnosis of corresponding biopsies was confirmed as above.

\section{Tube formation assay}

Ibidi $\mu$ slide angiogenesis slides (Ibidi, Germany) were coated with $10 \mu \mathrm{L}$ growth factor nutrient-reduced Matrigel (BD Biosciences, USA), and allowed to polymerase for $30 \mathrm{~min}$ at $37^{\circ} \mathrm{C}$. Cells were seeded in triplicate $\left(1.6 \times 10^{5}\right.$ cells/well) in complete DMEM and incubated at $37^{\circ} \mathrm{C}$. For MM and HUVEC co-culture, $8 \times 10^{5}$ cells from each cell type were combined and subsequently seeded at $1.6 \times 10^{5}$ cells/well onto Matrigel. Photos were taken at $1 \mathrm{~h}$ and $6 \mathrm{~h}$ on an Olympus IX71 Fluorescence Inverted Microscope (Olympus, Japan), and 'stitched' together using Adobe Photoshop (Adobe, USA).

\section{Immunofluorescent staining of tube formation assays}

Cultures were washed twice with PBS, and fixed for $30 \mathrm{~min}$ at $25^{\circ} \mathrm{C}$ with $10 \%$ buffered formalin. Blocking was performed using $10 \%$ normal goat serum for $90 \mathrm{~min}$, followed by addition of 1:100 primary antibody (Calretinin, 18-0211, Zymed, USA; CD31, JC/70A, M0823, Dako, Denmark) in 10\% normal goat serum. After $2 \mathrm{~h}$ incubation, wells were washed and incubated with secondary antibody for 2 h. Nuclei were counterstained with Hoechst 33342 (Bio-Rad, USA) for $30 \mathrm{~min}$ at $25^{\circ} \mathrm{C}$, then cells were washed and visualised with an Olympus IX71 Fluorescence Inverted Microscope.

\section{Animal model}

A heterotopic xenograft mouse model of MM was used to evaluate the origin of the vasculature in MM. Briefly, $1 \times 10^{6} \mathrm{NCI}-\mathrm{H} 226$ cells in PBS were injected subcutaneously into the hind flank of BALB/C nude mice $(n=6)$. Tumours were grown to $100 \mathrm{~mm}^{3}$ and animals euthanised by $\mathrm{CO}_{2}$ exposure (approved by Flinders University and Southern Adelaide Local Health Network Animal Welfare Committee).

\section{Immunohistochemistry}

All diagnostic immunohistochemical studies were performed by a clinical laboratory participating in a quality assurance program (QAP), using National Association of Testing Authorities (NATA)-approved procedures and with appropriate controls. For cell block preparation from cultured cells and effusions, cell pellets were resuspended in 1-2 drops of sheep plasma. One drop of thrombin was added and the mixture was allowed to set before immersion in $10 \%$ formalin. Graded concentrations of ethanol and isopropanol were applied before embedding in paraffin. Paraffin sections were cut at $4 \mu \mathrm{m}$, deparaffinised and quenched with $1 \% \mathrm{H}_{2} \mathrm{O}_{2}$. Cell block sections originating from cultured cells then underwent immunohistochemistry under the same conditions as clinical samples. For the human mitochondria immunohistochemistry, sections underwent EDTA retrieval and block with $10 \%$ normal goat serum (Sigma), before overnight incubation with 1:750 primary antibody MAB1273B (clone 113-1; Merck Millipore, USA).

\section{Human MM biopsy diagnosis}

Of the five biopsy cases reported in this study, two were identified among 18 in-house MMs of any histological subtype investigated at the Flinders Medical Centre (FMC) over a 15-month interval in 2015-2016; three cases represented referrals among a greater number of cases for most of which no paraffin embedded tissue was received. Diagnosis was established by light microscopy and positive immunohistochemical (IHC) labelling for epithelioid MM markers (CK5/6, calretinin, WT1, D2-40, thrombomodulin and HBME1), ${ }^{32,44}$ to exclude an epithelioid haemangioendothelioma (EHE) or an epithelioid angiosarcoma (ASa), ${ }^{45-47}$ supplemented by radiological demonstration that the tumour was serosa-based (pleural or, in one case, peritoneal), with no imaging evidence of any extraserosal tumour. ${ }^{32,34}$ One referred biopsy case was also studied by immunohistochemistry for glycophorin A as a marker for erythrocyte cell membranes. ${ }^{48}$ Another referral case was immunolabelled for TLE-1 (Transducin-like enhancer of split-1) and Erythroblast transformation specific related gene product (ERG; a marker for endothelial cells and some prostatic carcinomas ${ }^{49,50}$ ). Although TLE-1 labelling is demonstrable in about $90 \%$ of synovial sarcomas or more, it is also recorded in benign schwannian and solitary fibrous tumours, and up to about $37 \%$ of non-synovial sarcomas that include malignant peripheral nerve sheath cell tumours. ${ }^{51-53}$ Matsuyama et al. ${ }^{54}$ found TLE-1 expression in $>25 \%$ of cells in 20 of 29 mesotheliomas of all histological subtypes (about $69 \%$ ), and those authors ${ }^{54}$ concluded that TLE-1 expression had no or only limited value in the distinction between mesothelioma and synovial sarcoma. Some 'early' papers on VM included staining with periodic acid-Schiff (PAS) (plus CD31 immunohistochemistry ${ }^{10}$ ) or immunohistochemistry for laminin; however, PAS can stain epithelial, mesothelial ${ }^{55}$ or vascular basement membranes, and the same consideration applies to laminin immunohistochemistry, so that such markers do not per se distinguish between mesothelial and endothelial differentiation. ${ }^{56}$ Therefore, we have concentrated on CD31 positivity in combination with strong cytokeratin expression and mesothelial cell markers in step sections of the same areas of the same mesotheliomas.

\section{RESULTS}

\section{Mesothelioma cell lines and MM primary cells form tube-like interconnected networks in vitro under permissive conditions}

All MM cell lines tested (NCI-H226, NCI-H28, NCI-H2052, NCI-2452 and MSTO-211H) formed tube-like structures after $6 \mathrm{~h}$ incubation in vitro (Fig. $1 \mathrm{~B}-\mathrm{F}$ ) when cultured on reconstituted basement membrane (Matrigel) without growth factors. Likewise, all 12 primary MM cell samples obtained from pleural effusion fluids showed tube formation (Fig. 1G, representative of the seven epithelioid MMs tested; Fig. $1 \mathrm{H}$ the single sarcomatoid MM). The tubes formed by the MM cells were comparable to positive control tubes formed by bona fide vascular cells, HUVECs, under the same conditions (Fig. 1I). In contrast, mesothelial cells obtained from reactive effusions and the normal mesothelial Met5a cell line (Fig. 1A) did not form these networks under the same conditions. The tube-like structures did not label for CD31. Calretinin and cytokeratin expression were maintained in the cultured cells, including the single sarcomatoid MM.

\section{Mesothelioma primary cells and HUVECs form interconnected networks in vitro}

Primary MM cells incubated together with HUVECs formed interconnected networks consisting of both cell types, indicated by labelling of the mesothelial component for calretinin. There were no isolated islands of either cell type present, suggesting that vascular endothelial cells and MM tumour cells are capable of forming vascular mosaic networks (Fig. 1J). 

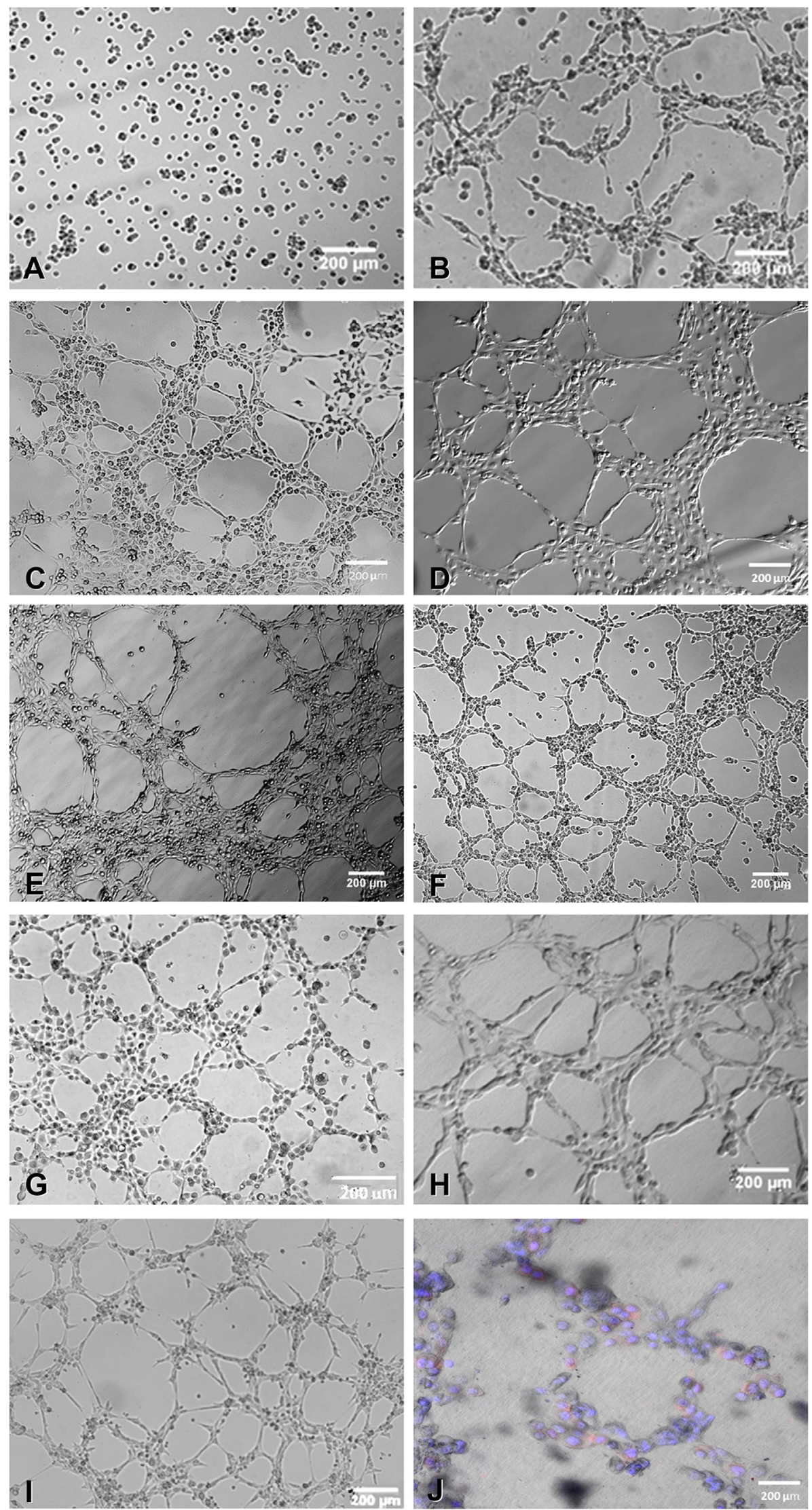

Fig. 1 MM cell lines and primary cells undergo vasculogenic mimicry in vitro. Cells were seeded $\left(1.6 \times 10^{5}\right.$ cells/well) onto Matrigel and incubated for 6 h. For coculture of HUVECs and MM, cells were labelled in situ with the anti-mesothelial antibody calretinin (red), and counterstained with Hoechst (blue). Photos were taken using an Olympus IX71 inverted fluorescence microscope fitted with $4 \times$ objective. The vasculogenic mimicry potential of MM cell lines was comparable to that of HUVECs, in contrast to the benign control cell line Met5a which did not exhibit tube formation under the same conditions. Co-cultured HUVEC and MM formed tubelike structures consisting of a mosaic of both cell types. (A) Met5a showing absence of tubal networks. (B) NCI-H28. (C) NCI-H226. (D) NCI-H2052. (E) NCI-H2452. (F) MSTO-211H. (G) Primary MM cells (epithelioid subtype). (H) Primary MM cells (sarcomatoid subtype). (I) HUVECs. (J) Primary MM cells co-cultured with HUVECs, labelled with the mesothelial marker calretinin (red), counterstained with Hoechst nuclear stain (blue). 


\section{The vasculature in a heterotopic MM xenograft model is partially of human origin}

Immunohistochemistry for human-specific mitochondria in sections of heterotopic xenografted $\mathrm{MM}$ in BALB/C nude mice confirmed that some vessels were of mouse origin, being lined with mouse endothelial cells, but labelling with the human mitochondria-specific antibody MAB1273B showed labelling of cells lining vessels containing red blood cells at the periphery of the tumour, ${ }^{57}$ indicative of VM in vivo (Fig. 2). Labelling for CD31 was apparent in all vascular structures, irrespective of human or mouse origin.

\section{Some biopsies of human MMs show clinical evidence of vascular mimicry}

As depicted in Fig. 3-6 and discussed in the corresponding legends, we found that in some biopsy samples of MMs, some cytokeratin-positive spindle and/or epithelioid cells formed vascular channels containing red blood cells, and the cells participating in vessel formation showed co-labelling for CD31 and cytokeratins, suggesting that VM is a significant feature in some MMs.

\section{DISCUSSION}

It was long thought that the microvasculature in malignant neoplasms represents proliferation of host micro-vessels driven by the secretion of angiogenic factors by the neoplastic cell population. A more complex picture has now emerged, beginning with the first report on VM by Maniotis et al. ${ }^{10}$ in 1999 . VM has now been recorded in a variety of human malignant neoplasms, including uveal and cutaneous melanoma, ${ }^{22}$ some breast carcinomas, ${ }^{20,58}$ hepatocellular carcinoma, ${ }^{21,23}$ gastric adenocarcinoma ${ }^{16}$ and, especially, glioblastoma. ${ }^{59-68}$ In this study, we adduce multifactorial evidence for VM ('angioblastic differentiation') in human MM, as demonstrated by: (1) the findings of 3-dimensional tubular networks of human MM cells in vitro, including all of the standard commercially available MM cell lines tested and 12 separate samples of primary MM cells harvested from pleural effusion fluids in biopsy-proven cases of MM, although no such networks were identified in cultures of benign reactive mesothelial cells; (2) the differential expression of a human mitochondrial marker in vascular structures when a standard human MM cell line (NCIH226) was xenotransplanted into a nude mouse; and (3) a few conventional biopsy samples of human MM, although
VM seems to be unrecognisable in most 'routine' biopsy cases.

It is important to note in this context that the formation of interconnecting networks occurred spontaneously when MM cells were cultured on reconstituted basement membrane (Matrigel) without growth factors, i.e., without added VEGF. Unlike conventional culture, this matrix simply allows threedimensional structures to form, but does not contain proangiogenic growth factors. However, MM cells themselves can secrete VEGF. It is unlikely that VM in MM coincides with epithelial to mesenchymal transition ${ }^{41}$ (previously shown to correlate with sarcomatoid change), because all primary MM cells tested were capable of VM in vitro, and only one sample was sarcomatoid in type (sarcomatoid MMs are notoriously paucicellular in effusion fluid). Rather, this may be an expression of the known pluripotency of mesothelial cells. This suggests that the positive labelling of tumour cells for endothelial markers in the biopsies represents an 'extreme' degree of endothelial differentiation in recognisable tumour cells, whereas in the majority of cases, those cells that have transitioned to vascular structures are simply not recognised as being neoplastic in character. The presence of human-derived vessels in a standard heterotopic xenograft MM model, where a MM tumour cell line (i.e., no possible contamination by human vascular cells) was injected also supports the notion that this may not be uncommon in MM. The labelling of all vessels for CD31 in the animal model also suggests that in biopsy samples VM may easily be overlooked, unless non-vascular tumour cells also express CD31. Labelling for CD31 is not necessary for MM cells to acquire a vascular phenotype, because the tube-like formations in vitro did not label for CD31.

The majority (67-93\%) of human MM samples label for thrombomodulin as well as cytokeratins, ${ }^{32,36-40}$ and this may be an indication of the ability to differentiate into vascular structures. Rare MMs also show labelling of tumour cells for CD $31,{ }^{69}$ and as discussed above, it is impossible to ascertain by CD31 labelling alone whether vessels are of tumour cell origin or stromal-derived microvasculature. Two "conventional' mesothelial markers - thrombomodulin (CD141) and D2-40 - are also endothelial markers, ${ }^{39,70,71}$ suggesting some plasticity in differentiation. There has been a previous report of vasculogenic mimicry in 'mesothelial sarcomas' 24 but this is not standard nomenclature, and that study did not specify the criteria used to make that diagnosis. ${ }^{24}$

Clearly, a major differential diagnostic consideration in the biopsy cases that we illustrate is MM with VM versus

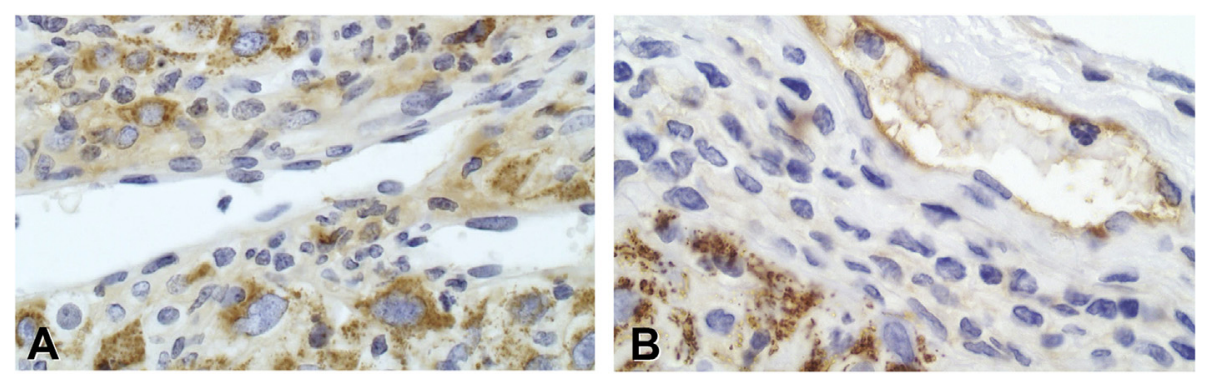

Fig. 2 Immunohistochemistry for human-derived vasculature in a heterotopic xenograft model. Human MM cells were grown in the flank of nude mice and the resultant tumour excised once it reached $100 \mathrm{~mm}^{3}$. The excised tumour was fixed in paraffin, and labelled with the human-specific antibody MAB1273B, which labels human mitochondria only (brown staining). (A) Vessel containing blood cells within the middle of the tumour is lined by mouse endothelial cells, unlabelled with MAB1273B. The surrounding human-derived tumour cells do label. (B) Vessel containing red blood cells with a lining of MAB1273B-labelled cells at the tumour periphery, adjacent mouse tissue. This indicates that tumour cells can generate their own vasculature. 

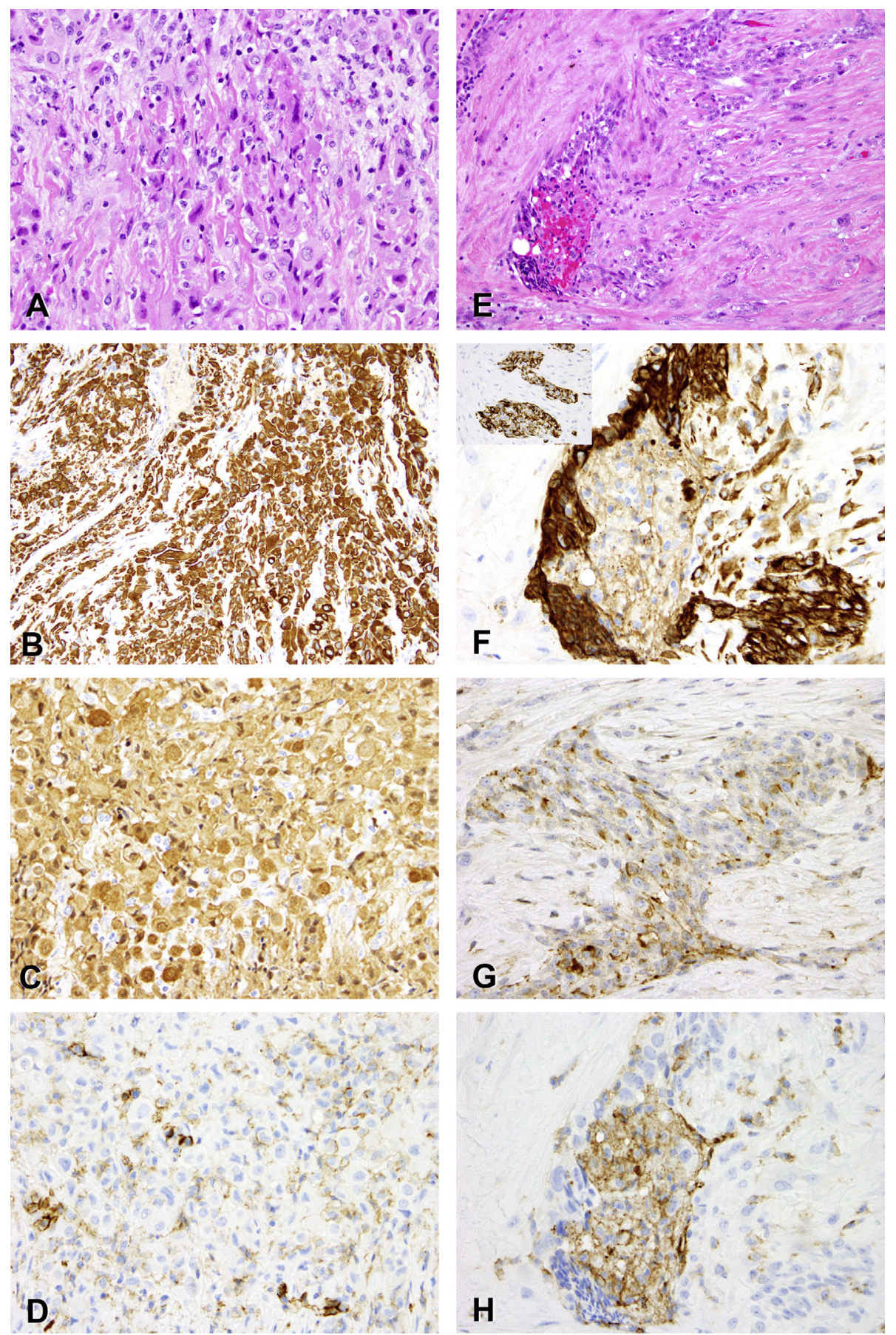

Fig. 3 (A-D) Pleural epithelioid MM biopsy from a 72-year-old woman with calcified pleural plaques whose husband had died from an asbestos-related disease. She also had a history of cutaneous melanoma, and immunohistochemistry revealed focal loss of BAP1 expression. The biopsy showed positive labelling for cytokeratin (CK) 8/18 as well as the mesothelial markers CK5/6, calretinin, WT1, D2-40, HBME-1 and thrombomodulin, whereas all carcinoma-related and melanoma markers were negative. The biopsy also showed positive labelling with/for CD31. (A) Histological appearances of the tumour. (B) AE1/AE3. (C) Step section from same area as A and B with calretinin expression. (D) Step section from same area as A-C with positive labelling of tumour cells for CD31, mainly linear. (E-H) Pleural biopsy from a 75year-old man. The epithelioid MM showed positive labelling with/for AE1/AE3, CK5/6, calretinin (focal), WT1 (weak to moderate), D2-40 (weak to moderate), HBME1, and thrombomodulin. The epithelioid cells comprising this MM also showed weak but definite labelling for CD31, although CD34 was negative. (E) Histological appearances of the tumour. (F) Step section of the area depicted above with positive labelling with AE1/AE3 and for CK5/6 (inset). (G) Step section of the same region as the two figures above with positive labelling for D2-40. (H) Step section of the same region as E with positive labelling for CD31.

pseudomesotheliomatous $\mathrm{ASa}^{72}$ or EHE. ${ }^{72}$ Serosal endothelial sarcomas are rare and reported cases of pleural ASa were defined by positive labelling for endothelial markers ${ }^{45,72-74}$ and negative mesothelial markers. ${ }^{45,47}$ Some reports on serosal $\mathrm{ASa} / \mathrm{EHE}$ do not include any data on labelling for mesothelial markers; ${ }^{45,73-76}$ two other publications recorded a negative result for the only mesothelial marker used, namely calretinin ${ }^{46}$ or HBME-1. ${ }^{45}$ Labelling for vimentin and cytokeratins may not reliably discriminate between $\mathrm{MM}$ and endothelial sarcomas. ${ }^{45}$ For these reasons, we have used multiple (six) MM markers-CK5/6, calretinin, WT1, D240, HBME-1 and thrombomodulin - and we have sought 


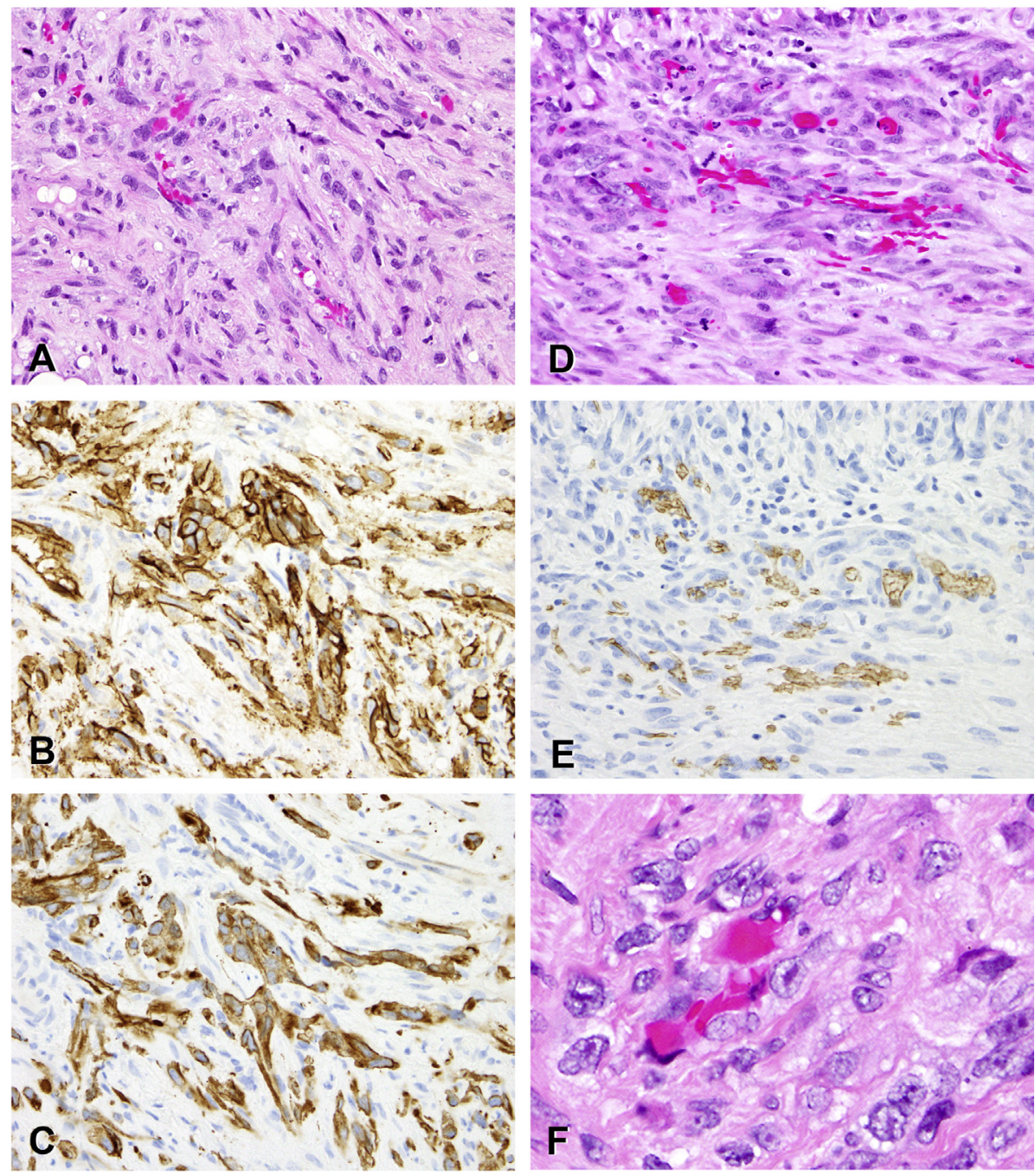

Fig. 4 Biopsy from a diffuse omental/peritoneal tumour in a 41-year-old woman, who presented with abdominal pain, ascites and weight loss. CT scans revealed no abnormality in the abdominal and pelvic viscera or lymphadenopathy. The biopsy showed a malignant and predominantly spindle-cell tumour. IHC studies revealed positive labelling with/for vimentin, AE1/AE3, CAM5.2 and CK7, but CK20 was negative. There was positive labelling for the mesothelial markers calretinin (focal), WT1 (focal), and thrombomodulin, as well as smooth muscle actin, and there was strong positive labelling for CD31, together with much weaker labelling for CD34 and factor VIII-related antigen. The label for BAP1 was positive. The preparation for Stat 6 was negative. The slides were reviewed by an expert in soft tissue tumour pathology, who favoured a diagnosis of a predominantly sarcomatoid MM with vasoformative features. ${ }^{69}$ (A) Microvascular channels with closely-associated pleomorphic and spindle-shaped tumour cells. (B) Step section of same area as A with positive labelling for CD31. (C) Step section of same area as A with positive labelling with AE1/AE3. (D) Predominantly sarcomatoid tissue with mitotic figures and pleomorphic tumour cells closely related to microvessels. (E) Same area as D, immunolabelled for glycophorin A to highlight cell membranes of intraluminal erythrocytes. ${ }^{48}$ (F) High magnification of vascular channel with associated pleomorphic tumour cells with indentation of erythrocytes.

multifactorial evidence for VM in MM (i.e., cell culture of $\mathrm{MM}$, and human mitochondrial immunohistochemistry in a MM xenograft). We consider those five clinical tumours to represent MM, based on co-expression of multiple mesothelial markers, areas of tubulopapillary architecture, and spindle-cell sarcomatoid areas in some tumours. The concept that MM can show specific patterns of mesenchymal differentiation (including heterologous sarcomatoid differentiation) may in part explain the readiness for differentiation as vessels under permissive conditions in vitro. The known pluripotency of normal mesothelial cells has resulted in their use for tissue engineering, including replacement of neuralcrest derived corneal endothelium. ${ }^{97}$ Our study has found that evidence of vascular differentiation in biopsies of MM varies greatly and Attanoos et al. ${ }^{73}$ found no CD31 expression in 92 mesotheliomas investigated, but all primary MM cells tested as part of our study were capable of VM in vitro.
Terada et $a l .{ }^{78}$ suggested their case of pericardial ASa might have represented a peculiar MM with abnormal differentiation along an angioblastic pathway, and in 2012 Klabatsa et $a l .{ }^{69}$ reported a diffuse pleural mesothelioma with epithelioid and angiosarcomatous components, which may correspond to our clinical cases: angioblastic differentiation may be analogous to heterologous osteochondroid differentiation in the sarcomatoid component of some MMs. ${ }^{35}$ Although CD31 can label macrophages, we consider the co-labelling for cytokeratins (and other more specific mesothelial markers in step sections the same cell clusters in our biopsy cases and labelling for ERG in one case) negates this explanation for the CD31 positivity.

We consider that VM is a property of MM: we are unable to estimate with any reasonable degree of precision the proportion of MMs that display immunohistochemical evidence of $\mathrm{VM}$ in the form of CD31 expression in human biopsy 

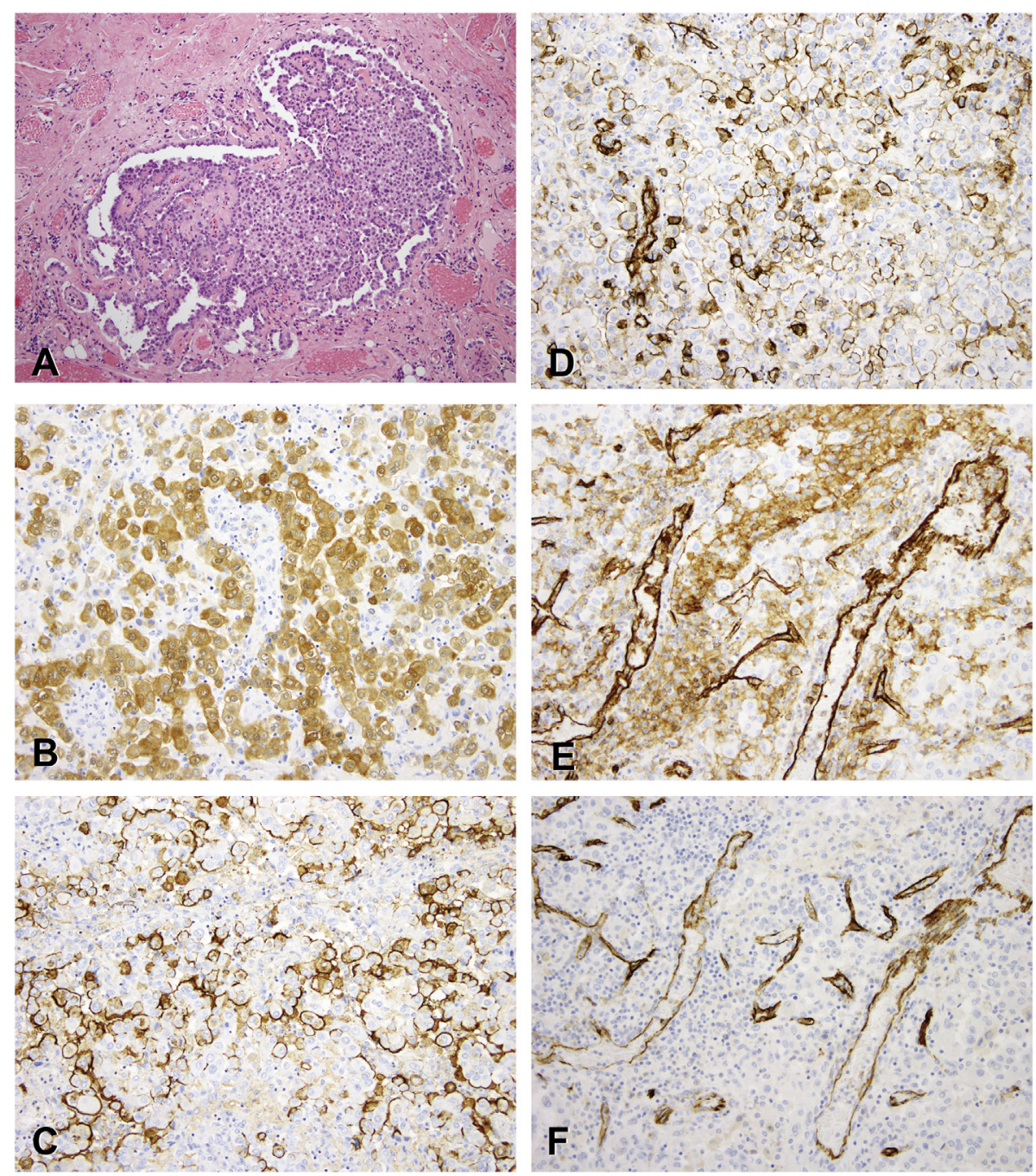

Fig. 5 Peritoneal mesothelioma from a 33-year-old woman treated by radical peritonectomy. Gross examination revealed multiple nodules of tan tumour tissue affecting the peritoneum and omentum. The MM showed positive IHC labelling with AE1/AE3 and for the mesothelial markers CK5/6, calretinin, WT1, D2-40 and HBME-1; there was positive labelling for BAP1 and EMA (linear), whereas all carcinoma-related markers were negative (CEA, CD15, BG8, CDX2, PAX8 and TTF-1). (A) Epithelioid/tubulopapillary architecture in tumour invading subcutaneous tissue at the umbilicus (H\&E). (B) Calretinin labelling. (C) Linear labelling for HBME-1. (D) Linear labelling for thrombomodulin (CD141). (E) CD31 labelling, with near-circumferential linear labelling of some individual tumour cells. (F) The CD34 preparation was negative in the tumour cells.

tissue (perhaps because it is unrecognisable as such in most biopsy samples), but the in vitro findings in our study point to VM as a routine occurrence in MM, with the capacity for anastomosis/mosaicism with host vasculature (Fig. 7), and VM was detectable not only in our MMs with sarcomatoid differentiation, but also in epithelioid MMs. We consider that CD31 labelling in otherwise typical MM samples may simply be an IHC marker for hybrid mesothelial $\rightarrow$ endothelial differentiation that is part of the pluripotency of MM (with epithelial to mesenchymal transition). Such EMT is at least latent in up to about $50 \%$ or more of epithelioid mesotheliomas, and it occurs in about $75 \%$ of biphasic MMs and about $85 \%$ of sarcomatoid MMs, ${ }^{79}$ as demonstrated by vimentincytokeratin co-synthesis: in their study of 326 sarcomatoid and desmoplastic MMs, Klebe et al. ${ }^{80}$ detected vimentin expression in $101 / 111$ cases $(91 \%)$, cytokeratins in 161/ $280(93 \%)$ and calretinin expression in $12 / 39$ cases $(31 \%)$. Alpha-smooth muscle actin (SMA) and desmin expression is detectable by immunohistochemistry in about $10 \%$ of biphasic MMs (and in a greater proportion of reactive mesothelial hyperplasias, up to about $85 \%){ }^{79,81}$ The exact mechanisms for such EMT in MM appear to be complex and are incompletely understood. Fassina et $a l .{ }^{41}$ studied N-cadherin, vimentin, SMA, Snail, Slug, Twist, ZEB1, ZEB2, S100A4, MMP2, and MMP9 from epithelioid to biphasic and sarcomatoid MM cell lines by immunohistochemistry and qRT-PCR, and in situ hybridisation. They found ectopic expression of miR-205 (a repressor of ZEB1 and ZEB2 expression) in a commercially available mesothelial cell line and in epithelioid and biphasic MM cell lines and it induced a significant reduction of ZEB1 and ZEB2, with inhibition of migration and invasion. In particular, miR205 was significantly down-regulated in biphasic and sarcomatoid MMs, correlating with a mesenchymal phenotype and more aggressive behaviour. De Reynies et al. ${ }^{42}$ investigated 38 primary pleural $\mathrm{MM}$ cell cultures by transcriptomic microarrays (discovery + extension series; $n=67$ ), and 108 frozen pleural MMs by qRT-PCR for gene mutations that included $B A P 1, C D K N 2 A, C D K N 2 B, N F 2$ and TP53; EMT markers were studied at mRNA and proteonomic levels. From these studies they divided their MMs into two groups: $\mathrm{C} 1$ and $\mathrm{C} 2$. All sarcomatoid/desmoplastic MMs fell into C2, 


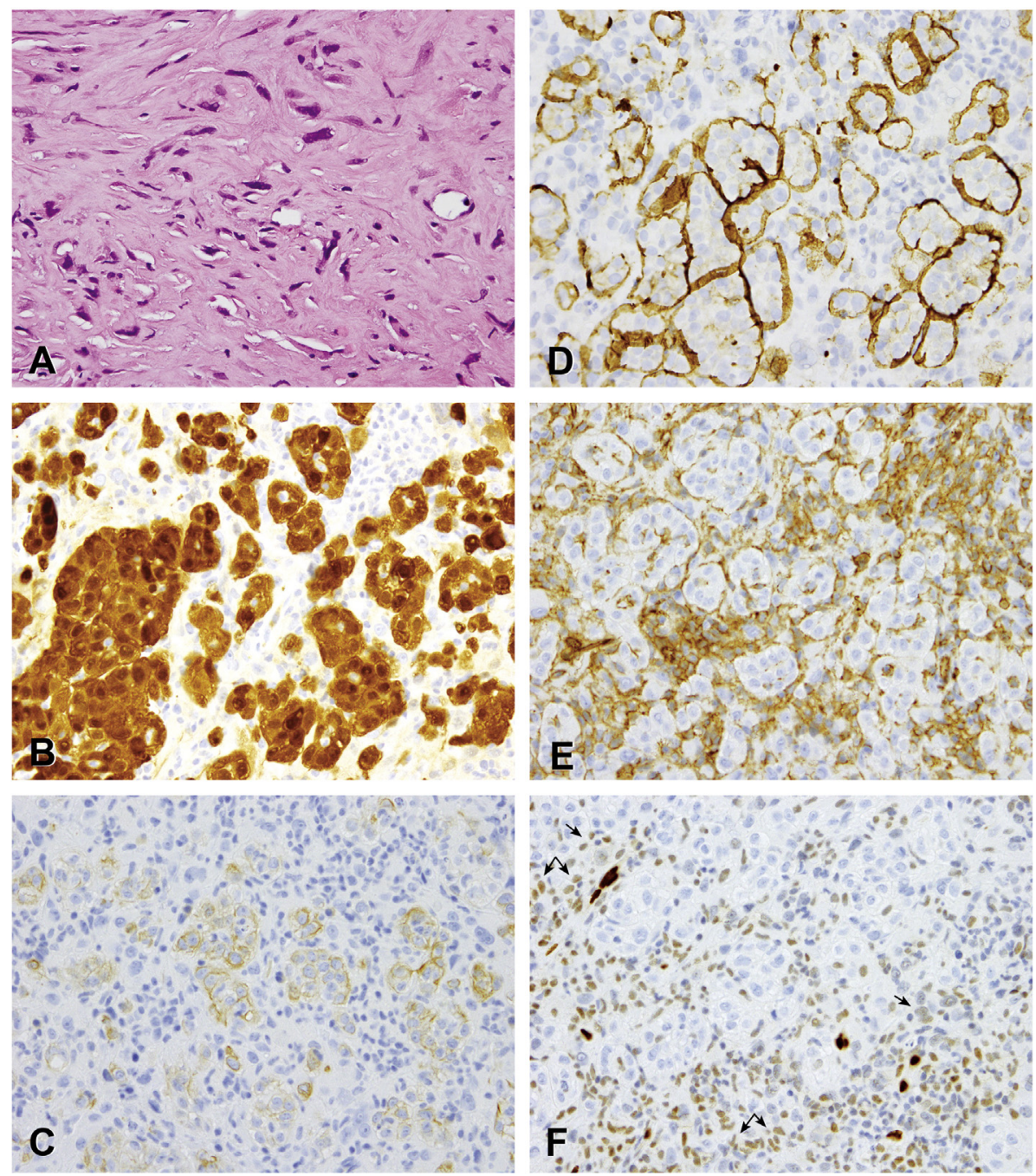

Fig. 6 Biopsies from a 63-year-old woman with a history of past occupational exposure to asbestos. She presented with a $100 \mathrm{~mm}$ mass lesion in the right anterosuperior mediastinum with evidence of chest wall invasion, accompanied by erosion of the second rib. Diagnoses of synovial sarcoma and leiomyosarcoma were considered [the tumour labelled for TLE- $1^{54}$ but FISH for $\mathrm{t}(\mathrm{X} ; 18)$ was negative]. Two biopsies were taken. The tumour in the second biopsy labelled with/for AE1/AE3 and CK5/6 (weak), D2-40, WT1 and HBME-1. Labelling was also observed for CD31 and another endothelial marker, ERG. A final diagnosis of mesothelioma was favoured and together the two biopsies showed a biphasic malignant MM, with desmoplastic features in the first biopsy (A). (B-E) Step sections from the same area of the second biopsy. (B) Calretinin labelling. (C) Weak labelling for CK5/6. (D) D2-40 positive labelling of tumour cells. (E) CD31 positive labelling of small blood vessels and in a linear pattern around tumour cells. (F) Labelling for ERG, in host blood vessels and with weaker labelling of tumour cell nuclei (arrows).

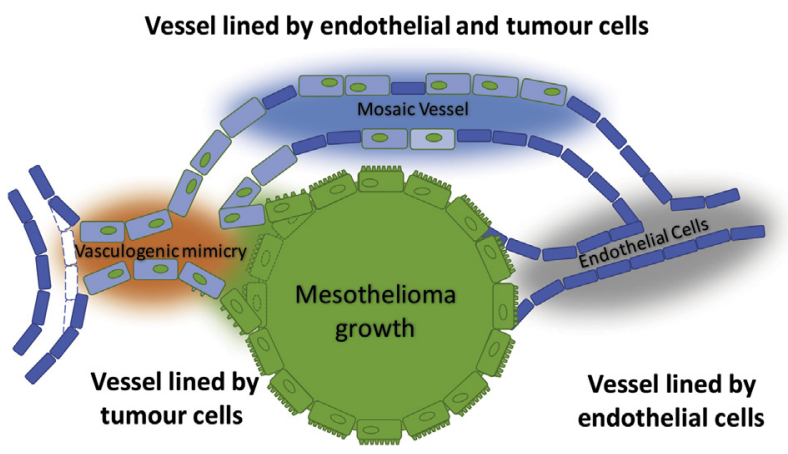

Fig. 7 The mechanisms of conventional angiogenesis, with endothelial-lined vessels supplying the growing tumour with blood. In contrast, in vascular mimicry, tumour cells arrange themselves to form a lumen that can carry blood, and assume some endothelial characteristics. These vessels could link into preexisting conventional vasculature, or alternatively, mosaic vessels that are lined by a mixture of tumour cells and endothelial cells could be formed. whereas the epithelioid and biphasic MMs fell into both $\mathrm{C} 1$ and $\mathrm{C} 2$, partly correlating with histological subtyping. Genes up-regulated during EMT (such as the adhesion molecules POSTN and VCAN or the transcription factors SANI2, TCF4 and $H M G A 2$ ) showed higher expression in $\mathrm{C} 2$ than $\mathrm{C} 1$, whereas genes down-regulated during EMT such as $\mathrm{CDH1}$ and $\mathrm{CDH} 3$ showed lower expression in $\mathrm{C} 2$ than $\mathrm{C} 1$. The prognosis for $\mathrm{C} 2$ was worse than for $\mathrm{C} 1$. In a further prospective study of VM in MMs, we hope to explore further the EMT pathways implicated in MMs with VM, by immunohistochemistry, and proteonomic and metabolomic analysis, together with studies for cancer stem cells in MM.

Acknowledgements: DWH and SK are indebted to Dr P.W. Allen for his review of the biopsy slides for Case 3, and to the pathologists within Australia who refer cases to us for opinion; all of the mesotheliomas comprising this study represent in-house or professional referrals. We also gratefully acknowledge Mr Jim Manavis for his assistance in human mitochondria immunohistochemistry techniques. 
Conflicts of interest and sources of funding: Funding was provided by the Flinders Medical Centre Foundation and the Faculty of Health Sciences of Flinders University. DWH and SK provide reports for the courts in Australia, at the request of both plaintiff and defendant lawyers, but declare no conflicts of interest concerning this study. All other authors state they have no conflicts of interest to disclose.

Address for correspondence: A/Prof Sonja Klebe, Department of Anatomical Pathology, Flinders University, Flinders Medical Centre, Bedford Park, SA 5042, Australia. E-mail: sonja.klebe@sa.gov.au

\section{References}

1. Gordon JR, Leigh J. Medicolegal aspects of the third wave of asbestosrelated disease in Australia. Med J Aust 2011; 195: 247-8.

2. Soeberg MJ, Creighton N, Currow DC, et al. Patterns in the incidence, mortality and survival of malignant pleural and peritoneal mesothelioma, New South Wales, 1972-2009. Aust NZ J Public Health 2016; 40 255-62.

3. Soeberg MJ, Leigh J, Driscoll T, et al. Incidence and survival trends for malignant pleural and peritoneal mesothelioma, Australia, 1982-2009. Occup Environ Med 2016; 73: 187-94.

4. Gatta G, Ciccolallo L, Kunkler I, et al. Survival from rare cancer in adults: a population-based study. Lancet Oncol 2006; 7: 132-40.

5. Edwards JG, Cox G, Andi A, et al. Angiogenesis is an independent prognostic factor in malignant mesothelioma. Br J Cancer 2001; 85: $863-8$.

6. Kumar-Singh S, Vermeulen PB, Weyler J, et al. Evaluation of tumour angiogenesis as a prognostic marker in malignant mesothelioma. J Pathol 1997; 182: 211-6.

7. Weyn B, Tjalma WA, Vermeylen P, et al. Determination of tumour prognosis based on angiogenesis-related vascular patterns measured by fractal and syntactic structure analysis. Clin Oncol (R Coll Radiol) 2004; 16: $307-16$.

8. Hirayama N, Tabata C, Tabata R, et al. Pleural effusion VEGF levels as a prognostic factor of malignant pleural mesothelioma. Respir Med 2011; 105: 137-42.

9. Kao SC, Harvie R, Paturi F, et al. The predictive role of serum VEGF in an advanced malignant mesothelioma patient cohort treated with thalidomide alone or combined with cisplatin/gemcitabine. Lung Cancer 2012; 75: 248-54

10. Maniotis AJ, Folberg R, Hess A, et al. Vascular channel formation by human melanoma cells in vivo and in vitro: vasculogenic mimicry. Am J Pathol 1999; 155: 739-52.

11. Shirakawa K, Kobayashi H, Heike Y, et al. Hemodynamics in vasculogenic mimicry and angiogenesis of inflammatory breast cancer xenograft. Cancer Res 2002; 62: 560-6.

12. Ruf W, Seftor EA, Petrovan RJ, et al. Differential role of tissue factor pathway inhibitors 1 and 2 in melanoma vasculogenic mimicry. Cancer Res 2003; 63: 5381-9.

13. Rybak SM, Sanovich E, Hollingshead MG, et al. "Vasocrine" formation of tumor cell-lined vascular spaces: implications for rational design of antiangiogenic therapies. Cancer Res 2003; 63: 2812-9.

14. Hillen F, Kaijzel EL, Castermans K, et al. A transgenic Tie2-GFP athymic mouse model; a tool for vascular biology in xenograft tumors. Biochem Biophys Res Commun 2008; 368: 364-7.

15. Dunleavey JM, Xiao L, Thompson J, et al. Vascular channels formed by subpopulations of PECAM1+ melanoma cells. Nat Commun 2014; 5: 5200.

16. Li M, Gu Y, Zhang Z, et al. Vasculogenic mimicry: a new prognostic sign of gastric adenocarcinoma. Pathol Oncol Res 2010; 16: 259-66.

17. Hendrix MJ, Seftor EA, Hess AR, Seftor RE. Vasculogenic mimicry and tumour-cell plasticity: lessons from melanoma. Nat Rev Cancer 2003; 3 $411-21$.

18. Kirschmann DA, Seftor EA, Hardy KM, et al. Molecular pathways: vasculogenic mimicry in tumor cells: diagnostic and therapeutic implications. Clin Cancer Res 2012; 18: 2726-32.

19. Cao Z, Bao M, Miele L, et al. Tumour vasculogenic mimicry is associated with poor prognosis of human cancer patients: a systemic review and meta-analysis. Eur J Cancer 2013; 49: 3914-23.

20. Liu T, Sun B, Zhao X, et al. OCT4 expression and vasculogenic mimicry formation positively correlate with poor prognosis in human breast cancer. Int J Mol Sci 2014; 15: 19634-49.

21. Zhu MS, Xu LB, Zeng H, et al. Association of Notch1 with vasculogenic mimicry in human hepatocellular carcinoma cell lines. Int J Clin Exp Pathol 2014; 7: 5782-91.
22. Xia Y, Cai XY, Fan JQ, et al. Rho Kinase inhibitor Fasudil suppresses the vasculogenic mimicry of B16 mouse melanoma cells both in vitro and in vivo. Mol Cancer Ther 2015; 14: 1582-90.

23. Yang Z, Sun B, Zhao X, et al. Erythropoietin and erythropoietin receptor in hepatocellular carcinoma: correlation with vasculogenic mimicry and poor prognosis. Int J Clin Exp Pathol 2015; 8: 4033-43.

24. Sun B, Zhang S, Zhao X, et al. Vasculogenic mimicry is associated with poor survival in patients with mesothelial sarcomas and alveolar rhabdomyosarcomas. Int J Oncol 2004; 25: 1609-14.

25. Ohta Y, Shridhar V, Bright RK, et al. VEGF and VEGF type C play an important role in angiogenesis and lymphangiogenesis in human malignant mesothelioma tumours. Br J Cancer 1999; 81: 54-61.

26. Hooper CE, Elvers KT, Welsh GI, et al. VEGF and sVEGFR-1 in malignant pleural effusions: Association with survival and pleurodesis outcomes. Lung Cancer 2012; 77: 443-9.

27. Zebrowski BK, Yano S, Liu W, et al. Vascular endothelial growth factor levels and induction of permeability in malignant pleural effusions. Clin Cancer Res 1999; 5: 3364-8.

28. Konig J, Tolnay E, Wiethege T, Muller K. Co-expression of vascular endothelial growth factor and its receptor flt- 1 in malignant pleural mesothelioma. Respiration 2000; 67: 36-40.

29. Strizzi L, Catalano A, Vianale G, et al. Vascular endothelial growth factor is an autocrine growth factor in human malignant mesothelioma. J Pathol 2001; 193: 468-75.

30. Li Q, Yano S, Ogino H, et al. The therapeutic efficacy of anti vascular endothelial growth factor antibody, bevacizumab, and pemetrexed against orthotopically implanted human pleural mesothelioma cells in severe combined immunodeficient mice. Clin Cancer Res 2007; 13: $5918-25$.

31. Henderson DW, Shilkin KB, Whitaker D, et al. The pathology of mesothelioma, including immunohistology and ultrastructure. In Henderson DW, Shilkin KB, Langlois SL, Whitaker D, editors. Malignant Mesothelioma. New York: Hemisphere, 1992; 69-139.

32. Hammar SP, Henderson DW, Klebe S, Dodson RF. Neoplasms of the pleura. In: Tomashefski JF Jr, editor. Dail and Hammar's Pulmonary Pathology. 3rd ed. Vol 2. New York: Springer, 2008; 558-734.

33. Husain AN, Colby T, Ordonez N, et al. Guidelines for Pathologic Diagnosis of Malignant Mesothelioma: 2012 Update of the Consensus Statement from the International Mesothelioma Interest Group. Arch Pathol Lab Med 2012; 136: 1-21.

34. Pavlisko EN, Sporn TA. Mesothelioma. In: Oury TD, Sporn TA, Roggli VL, editors. Pathology of Asbestos-Associated Diseases. 3rd ed. New York: Springer, 2014; 81-140.

35. Klebe S, Mahar A. Henderson DW, Roggli VL. Malignant mesothelioma with heterologous elements: clinicopathological correlation of 27 cases and literature review. Mod Pathol 2008; 21: 1084-94.

36. Kennedy AD, King G, Kerr KM. HBME-1 and antithrombomodulin in the differential diagnosis of malignant mesothelioma of pleura. $J$ Clin Pathol 1997; 50: 859-62.

37. Ordonez NG. Value of thrombomodulin immunostaining in the diagnosis of mesothelioma. Histopathology 1997; 31: 25-30.

38. Fetsch PA, Abati A, Hijazi YM. Utility of the antibodies CA 19-9, HBME-1, and thrombomodulin in the diagnosis of malignant mesothelioma and adenocarcinoma in cytology. Cancer 1998; 84: 101-8.

39. Cury PM, Butcher DN, Fisher C, et al. Value of the mesotheliumassociated antibodies thrombomodulin, cytokeratin 5/6, calretinin, and $\mathrm{CD} 44 \mathrm{H}$ in distinguishing epithelioid pleural mesothelioma from adenocarcinoma metastatic to the pleura. Mod Pathol 2000; 13: 107-12.

40. Comin CE, Novelli L, Boddi V, et al. Calretinin, thrombomodulin, CEA, and CD15: a useful combination of immunohistochemical markers for differentiating pleural epithelial mesothelioma from peripheral pulmonary adenocarcinoma. Hum Pathol 2001; 32: 529-36.

41. Fassina A, Cappellesso R, Guzzardo V, et al. Epithelial-mesenchymal transition in malignant mesothelioma. Mod Pathol 2012; 25: 86-99.

42. de Reynies A, Jaurand MC, Renier A, et al. Molecular classification of malignant pleural mesothelioma: identification of a poor prognosis subgroup linked to the epithelial-to-mesenchymal transition. Clin Cancer Res 2014; 20: 1323-34.

43. Litwin M, Clark K, Noack L, et al. Novel cytokine-independent induction of endothelial adhesion molecules regulated by platelet/endothelial cell adhesion molecule (CD31). J Cell Biol 1997; 139: 219-28.

44. Galateau-Salle F, Churg A, Roggli V, et al. The 2015 World Health Organization Classification of Tumors of the Pleura: Advances since the 2004 Classification. J Thorac Oncol 2016; 11: 142-54.

45. Lin BT, Colby T, Gown AM, et al. Malignant vascular tumors of the serous membranes mimicking mesothelioma. A report of 14 cases. Am J Surg Pathol 1996; 20: 1431-9.

46. Del Frate C, Mortele K, Zanardi R, et al. Pseudomesotheliomatous angiosarcoma of the chest wall and pleura. J Thorac Imaging 2003; 18: $200-3$. 
47. Kao YC, Chow JM, Wang KM, et al. Primary pleural angiosarcoma as a mimicker of mesothelioma: a case report. Diagn Pathol 2011; 6: 130 .

48. Rehg JE, Bush D, Ward JM. The utility of immunohistochemistry for the identification of hematopoietic and lymphoid cells in normal tissue and interpretation of proliferative and inflammatory lesions of mice and rats. Toxicol Pathol 2012; 40: 345-74.

49. Miettinen M, Wang ZF, Paetau A, et al. ERG transcription factor as an immunohistochemical marker for vascular endothelial tumors and prostatic carcinoma. Am J Surg Pathol 2011; 35: 432-41.

50. Sullivan HC, Edgar MA, Cohen C, et al. The utility of ERG, CD31 and CD34 in the cytological diagnosis of angiosarcoma: an analysis of 25 cases. J Clin Pathol 2015; 68: 44-50.

51. Kosemehmetoglu K, Vrana JA, Folpe AL. TLE1 expression is not specific for synovial sarcoma: a whole section study of 163 soft tissue and bone neoplasms. Mod Pathol 2009; 22: 872-8.

52. Rekhi B, Basak R, Desai SB, Jambhekar NA. Immunohistochemical validation of TLE1, a novel marker, for synovial sarcomas. Indian J Med Res 2012; 136: 766-75.

53. Lin G, Doyle LA. An update on the application of newly described immunohistochemical markers in soft tissue pathology. Arch Pathol Lab Med 2015; 139: 106-21.

54. Matsuyama A, Hisaoka M, Iwasaki M, et al. TLE1 expression in malignant mesothelioma. Virchows Arch 2010; 457: 577-83.

55. Adams SA, Sherwood AJ, Smith ME. Malignant mesothelioma: PASdiastase positivity and inversion of polarity in intravascular tumour. Histopathology 2002; 41: 260-2.

56. Kallianpur AR, Carstens PH, Liotta LA, et al. Immunoreactivity in malignant mesotheliomas with antibodies to basement membrane components and their receptors. Mod Pathol 1990; 3: 11-8.

57. Lange T, Ullrich S, Muller I, et al. Human prostate cancer in a clinically relevant xenograft mouse model: identification of beta(1,6)-branched oligosaccharides as a marker of tumor progression. Clin Cancer Res 2012; 18: 1364-73.

58. Yao N, Ren K, Jiang C, et al. Combretastatin A4 phosphate treatment induces vasculogenic mimicry formation of W256 breast carcinoma tumor in vitro and in vivo. Tumour Biol 2015; 36: 8499-510.

59. Yue WY, Chen ZP. Does vasculogenic mimicry exist in astrocytoma? J Histochem Cytochem 2005; 53: 997-1002.

60. El Hallani S, Boisselier B, Peglion F, et al. A new alternative mechanism in glioblastoma vascularization: tubular vasculogenic mimicry. Brain 2010; 133: 973-82.

61. Ricci-Vitiani L, Pallini R, Biffoni M, et al. Tumour vascularization via endothelial differentiation of glioblastoma stem-like cells. Nature 2010; 468: 824-8

62. Chiao MT, Yang YC, Cheng WY, et al. CD133+ glioblastoma stem-like cells induce vascular mimicry in vivo. Curr Neurovasc Res 2011; 8: 210-9.

63. Francescone R, Scully S, Bentley B, et al. Glioblastoma-derived tumor cells induce vasculogenic mimicry through Flk-1 protein activation. J Biol Chem 2012; 287: 24821-31.
64. Scully S, Francescone R, Faibish M, et al. Transdifferentiation of glioblastoma stem-like cells into mural cells drives vasculogenic mimicry in glioblastomas. $J$ Neurosci 2012; 32: 12950-60.

65. Mao XG, Xue XY, Wang L, et al. CDH5 is specifically activated in glioblastoma stemlike cells and contributes to vasculogenic mimicry induced by hypoxia. Neuro Oncol 2013; 15: 865-79.

66. Wang SY, Ke YQ, Lu GH, et al. Vasculogenic mimicry is a prognostic factor for postoperative survival in patients with glioblastoma. J Neurooncol 2013; 112: 339-45.

67. Mao JM, Liu J, Guo G, et al. Glioblastoma vasculogenic mimicry: signaling pathways progression and potential anti-angiogenesis targets. Biomark Res 2015; 3: 8

68. Shao R, Taylor SL, Oh DS, Schwartz LM. Vascular heterogeneity and targeting: the role of YKL-40 in glioblastoma vascularization. Oncotarget 2015; 6: 40507-18.

69. Klabatsa A, Nicholson AG, Dulay K, et al. Diffuse pleural mesothelioma with epithelioid and angiosarcomatous components - a hitherto undescribed pattern of differentiation. Histopathology 2012; 60: 1164-6.

70. Nakamura Y, Kanemura Y, Yamada T, et al. D2-40 antibody immunoreactivity in developing human brain, brain tumors and cultured neural cells. Mod Pathol 2006; 19: 974-85.

71. Kalof AN, Cooper K. D2-40 immunohistochemistry - so far! Adv Anat Pathol 2009; 16: 62-4.

72. Zhang PJ, Livolsi VA, Brooks JJ. Malignant epithelioid vascular tumors of the pleura: report of a series and literature review. Hum Pathol 2000 31: $29-34$.

73. Attanoos RL, Suvarna SK, Rhead E, et al. Malignant vascular tumours of the pleura in "asbestos" workers and endothelial differentiation in malignant mesothelioma. Thorax 2000; 55: 860-3.

74. Roh MS, Seo JY, Hong SH. Epithelioid angiosarcoma of the pleura: case report. J Korean Med Sci 2001; 16: 792-5.

75. McCaughey WTE, Dardick I, Barr JR. Angiosarcoma of serous membranes. Arch Pathol Lab Med 1983; 107: 304-7.

76. Falconieri G, Bussani R, Mirra M, Zanella M. Pseudomesotheliomatous angiosarcoma: a pleuropulmonary lesion simulating malignant pleural mesothelioma. Histopathology 1997; 30: 419-24.

77. Lachaud CC, Soria F, Escacena N, et al. Mesothelial cells: a cellula surrogate for tissue engineering of corneal endothelium. Invest Ophthalmol Vis Sci 2014; 55: 5967-78.

78. Terada T, Nakanuma Y, Matsubara T, Suematsu T. An autopsy case of primary angiosarcoma of the pericardium mimicking malignant mesothelioma. Acta Pathol Jpn 1988; 38: 1345-51.

79. Mayall FG, Goddard H, Gibbs AR. Intermediate filament expression in mesotheliomas: Leiomyoid mesotheliomas are not uncommon. Histopathology 1992; 21: 453-7.

80. Klebe S, Brownlee NA, Mahar A, et al. Sarcomatoid mesothelioma: clinical-pathologic correlation of 326 cases. Mod Pathol 2010; 23: 470-9.

81. Attanoos RL, Griffin A, Gibbs AR. The use of immunohistochemistry in distinguishing reactive from neoplastic mesothelium. A novel use for desmin and comparative evaluation with epithelial membrane antigen, p53, platelet-derived growth factor-receptor, P-glycoprotein and Bcl-2. Histopathology 2003; 43: 231-8. 